\title{
An analytical model of mountain wave drag for wind profiles with shear and curvature
}

Article

Accepted Version

Teixeira, M. A. C., Miranda, P. M. A. and Valente, M. A. (2004) An analytical model of mountain wave drag for wind profiles with shear and curvature. Journal of the Atmospheric Sciences, 61 (9). pp. 1040-1054. ISSN 1520-0469 doi: https://doi.org/10.1175/15200469(2004)061<1040:AAMOMW>2.0.CO;2 Available at https://centaur.reading.ac.uk/29254/

It is advisable to refer to the publisher's version if you intend to cite from the work. See Guidance on citing.

To link to this article DOI: http://dx.doi.org/10.1175/1520-

0469(2004)061<1040:AAMOMW>2.0.CO;2

Publisher: American Meteorological Society

All outputs in CentAUR are protected by Intellectual Property Rights law, including copyright law. Copyright and IPR is retained by the creators or other copyright holders. Terms and conditions for use of this material are defined in the End User Agreement. 


\section{CentAUR}

Central Archive at the University of Reading

Reading's research outputs online 


\title{
An Analytical Model of Mountain Wave Drag for Wind Profiles with Shear and Curvature
}

\author{
Miguel A. C. Teixeira, Pedro M. A. Miranda, and Maria Antónia Valente \\ Centro de Geofísica, and Department of Physics, University of Lisbon, Lisbon, Portugal
}

(Manuscript received 10 March 2003, in final form 15 October 2003)

\begin{abstract}
An analytical model is developed to predict the surface drag exerted by internal gravity waves on an isolated axisymmetric mountain over which there is a stratified flow with a velocity profile that varies relatively slowly with height. The model is linear with respect to the perturbations induced by the mountain, and solves the Taylor-Goldstein equation with variable coefficients using a Wentzel-Kramers-Brillouin (WKB) approximation, formally valid for high Richardson numbers, Ri. The WKB solution is extended to a higher order than in previous studies, enabling a rigorous treatment of the effects of shear and curvature of the wind profile on the surface drag. In the hydrostatic approximation, closed formulas for the drag are derived for generic wind profiles, where the relative magnitude of the corrections to the leading-order drag (valid for a constant wind profile) does not depend on the detailed shape of the orography. The drag is found to vary proportionally to $\mathrm{Ri}^{-1}$, decreasing as Ri decreases for a wind that varies linearly with height, and increasing as Ri decreases for a wind that rotates with height maintaining its magnitude. In these two cases the surface drag is predicted to be aligned with the surface wind. When one of the wind components varies linearly with height and the other is constant, the surface drag is misaligned with the surface wind, especially for relatively small Ri. All these results are shown to be in fairly good agreement with numerical simulations of mesoscale nonhydrostatic models, for high and even moderate values of Ri.
\end{abstract}

\section{Introduction}

One of the basic effects of subgrid-scale orography that must be parameterized in large-scale atmospheric models is orographic gravity wave drag. This force arises in stratified flow over mountains due to the upwindleeward asymmetry of the pressure perturbation associated with stationary internal gravity waves generated by the mountains. With the same value and opposite direction to the drag exerted on the mountains, there is a reaction force acting on the atmosphere that tends to decelerate the flow. When integrated over the main mountain ranges, this force has an important impact on the global atmospheric circulation (McFarlane 1987). In order to develop physically sound drag parameterizations, it is necessary to know how the surface drag varies with the characteristics of the flow: the shape of the orography, its height and width, the stratification of the incoming flow, its velocity, and the associated velocity gradients.

As pointed out by Shutts (1995), surprisingly few studies exist that treat the effect of shear on mountain waves. Until now, most of these studies have either used

Corresponding author address: Miguel A. C. Teixeira, Centro de Geofísica da Universidade de Lisboa, Edifício C8, Campo Grande, 1749-016 Lisbon, Portugal.

E-mail: teixeira@fc.ul.pt numerical models or highly idealized analytical models, where the equations of motion are linearized with respect to the perturbations induced by the orography. A notable exception to this linearization is the model developed by Long (1953) where the equation for the streamline displacement is linear, while being valid for waves of arbitrary amplitude. Unfortunately, the validity of Long's solution, which has been used by Huppert and Miles (1969), Lilly and Klemp (1979), Smith (1985), and Durran (1992), is limited to unperturbed flows with constant velocity and 2D orography. Studies using numerical models often focus on the nonlinear regimes that cannot be simulated accurately using the linear approximation, such as resonance (Clark and Peltier 1984; Bacmeister and Pierrehumbert 1988; Scinocca and Peltier 1991; Miranda and Valente 1997) or lee vortices (Smolarkiewicz and Rotunno 1989). In these cases, the effect of shear cannot be easily separated from nonlinear effects.

Despite being limited in their application to flows over gentle orography, linear models are particularly useful for studying the dependence of the drag force on the parameters of the flow in an exhaustive way, because they have analytical solutions. A linear model has been used, for example, by Broad (1995), to derive a quite general extension to 3D of the Eliassen-Palm (EP) theorem. 
However, linear models also have the important limitation that closed formulas for the drag can only be obtained for a small set of orography shapes and highly simplified wind profiles. Analytical expressions for the surface drag have been obtained, in the hydrostatic approximation, for flow over bell-shaped ridges or isolated mountains, in the cases of a constant wind (Smith 1979, 1980; Phillips 1984), a wind that varies linearly with height (Smith 1986; Keller 1994; Grubišić and Smolarkiewicz 1997), and a wind profile with directional shear where one of the velocity components varies linearly and the other is constant (Shutts 1995; Shutts and Gadian 1999). The analytical resolution of the equations of motion is practically limited to these simple types of flow, and consideration of more complicated velocity profiles requires the use of approximate methods. One such method is the Wentzel-Kramers-Brillouin (WKB) approximation.

The WKB approximation is applicable to waves propagating in slowly varying media, and has been used by Grisogono (1994) to calculate the surface drag for unidirectional flow with a hyperbolic-tangent profile over a Gaussian ridge. The expression for the surface drag derived by Grisogono does not depend on the first derivative of the velocity, and this is clearly incorrect, as the studies of Smith (1986) and Grubišić and Smolarkiewicz (1997) have shown, respectively, for flow over a ridge and flow over an isolated mountain. In the studies of Shutts (1995) and Shutts and Gadian (1999), the WKB approximation has also been used to determine the vertical momentum flux in an atmosphere with weak shear. But the expression obtained by them for the surface drag is equal to that valid for an atmosphere with constant wind. An aim of this study is to overcome these limitations and present a model based on the WKB approximation that is totally consistent and capable of reproducing the correct asymptotic behavior of the surface drag displayed by previous exact analyses.

The effect of shear on the drag is reflected essentially through a dependence on the Richardson number of the flow, Ri. This study was motivated in part by the numerical results of Grubišić and Smolarkiewicz (1997) and of Valente (2000), which show that the surface drag displays totally different behaviors for a velocity profile with a linear variation and for a wind that rotates with height, decreasing as $\mathrm{Ri}$ decreases in the first case and increasing as Ri decreases in the second. Another aim of the present study is to present a framework from which expressions for the drag can be obtained for more general wind profiles, therefore clarifying these differences in behavior.

In this study, an improved linear solution for the surface gravity wave drag on an isolated mountain is developed, assuming that the velocity profile, and hence the vertical wavenumber of the waves varies slowly in the vertical. Adopting a WKB approximation, the vertical wavenumber of the internal gravity waves is expanded as a power series of a small parameter $\varepsilon$ in- versely proportional to the square root of the Richardson number. Unlike in the WKB treatments of Grisogono (1994) and Shutts (1995), this expansion is extended here to second order in $\varepsilon$, with the consequence that the first derivative of the velocity profile is now correctly predicted to have an impact on the drag, and the correction due to the curvature of the velocity profile is smaller than predicted by the approach of Grisogono, in better agreement with data. Although the WKB approximation is formally valid for high Richardson numbers, it will be seen that the model reproduces reasonably well the results of nonhydrostatic mesoscale numerical models not only for high but also for Richardson numbers of order one, for the three simple flows used as test cases.

This paper is organized as follows. Section 2 presents the theoretical model used in this study. The model is derived for nonhydrostatic conditions but is then simplified for hydrostatic flow. Section 3 presents the results, where the model is tested for three idealized flows. Finally, section 4 contains the main conclusions of this study.

\section{A second-order WKB linear model}

An analytical model is developed here to calculate the surface drag force produced by orographic internal gravity waves generated in a flow with constant BruntVäisälä frequency $N$ over an isolated mountain of height $h_{0}$ and half-width $a$. The model is linear, and hence valid for $\hat{h}=N h_{0} /\left|\mathbf{U}_{0}\right| \ll 1$, where $\mathbf{U}_{0}=\left(U_{0}, V_{0}\right)$ is the surface wind, but the wind velocity may nevertheless vary with height in a complicated way, provided that it varies relatively slowly. This variation makes the equation that governs the vertical structure of the mountain waves have variable coefficients, which in general would prevent obtaining an analytical solution. Here, this problem will be overcome using the WKB approximation (Bender and Orszag 1999). As usual in linear models, the flow is assumed to be steady and the equations of motion with the Boussinesq approximation are linearized with respect to the perturbations induced by the mountain, yielding

$$
\begin{aligned}
U \frac{\partial u}{\partial x}+V \frac{\partial u}{\partial y}+w \frac{d U}{d z} & =-\frac{1}{\rho_{0}} \frac{\partial p}{\partial x} \\
U \frac{\partial v}{\partial x}+V \frac{\partial v}{\partial y}+w \frac{d V}{d z} & =-\frac{1}{\rho_{0}} \frac{\partial p}{\partial y} \\
U \frac{\partial w}{\partial x}+V \frac{\partial w}{\partial y} & =-\frac{1}{\rho_{0}} \frac{\partial p}{\partial z}+g \frac{\theta}{\theta_{0}}, \\
U \frac{\partial \theta}{\partial x}+V \frac{\partial \theta}{\partial y}+w \frac{d \theta_{0}}{d z} & =0 \\
\frac{\partial u}{\partial x}+\frac{\partial v}{\partial y}+\frac{\partial w}{\partial z} & =0
\end{aligned}
$$

In this equation set it was assumed that the flow is 
inviscid and steady and that the earth's rotation is not important. The unperturbed wind velocity is $\mathbf{U}=[U(z)$, $V(z), 0]$ (which is horizontally uniform but varies with height $), \mathbf{u}=(u, v, w)$ is the velocity perturbation induced by the mountain, $\rho_{0}$ and $\theta_{0}$ are the unperturbed density and potential temperature, $g$ is the acceleration of gravity, and $p$ and $\theta$ are the perturbations of pressure and potential temperature.

By standard procedures, differentiating the equations of this set several times and combining them in order to eliminate the dependent variables, a single equation for the vertical velocity perturbation $w$ is obtained:

$$
\begin{gathered}
\left(U \frac{\partial}{\partial x}+V \frac{\partial}{\partial y}\right)^{2} \nabla^{2} w-\left(\frac{d^{2} U}{d z^{2}} \frac{\partial}{\partial x}+\frac{d^{2} V}{d z^{2}} \frac{\partial}{\partial y}\right)\left(U \frac{\partial w}{\partial x}+V \frac{\partial w}{\partial y}\right) \\
+N^{2}\left(\frac{\partial^{2} w}{\partial x^{2}}+\frac{\partial^{2} w}{\partial y^{2}}\right)=0
\end{gathered}
$$

where $N=\left[\left(g / \theta_{0}\right) d \theta_{0} / d z\right]^{1 / 2}$ is the Brunt-Väisälä frequency of the reference state. This is subject to the boundary condition

$$
w(z=0)=U(z=0) \frac{\partial \eta}{\partial x}+V(z=0) \frac{\partial \eta}{\partial y},
$$

where $\eta(x, y)$ is the surface elevation, and the additional condition that the wave energy radiates upward.

Differentiating (1) with respect to $x$ and (2) with respect to $y$, adding them and using mass conservation (5) yields an equation for the pressure perturbation:

$$
\begin{aligned}
\frac{\partial^{2} p}{\partial x^{2}} & +\frac{\partial^{2} p}{\partial y^{2}} \\
& =\rho_{0}\left(U \frac{\partial}{\partial x} \frac{\partial w}{\partial z}+V \frac{\partial}{\partial y} \frac{\partial w}{\partial z}-\frac{d U}{d z} \frac{\partial w}{\partial x}-\frac{d V}{d z} \frac{\partial w}{\partial y}\right) .
\end{aligned}
$$

In the linear approximation the pressure drag on orography is given by

$$
\mathbf{D}=\int_{-\infty}^{+\infty} \int_{-\infty}^{+\infty} p(z=0) \boldsymbol{\nabla}_{H} \eta d x d y,
$$

where $\boldsymbol{\nabla}_{H}=(\partial / \partial x, \partial / \partial y, 0)$ is the horizontal gradient operator. To determine the gravity wave drag in the present model, one must first solve (6), then use the solution for $w$ in (8) to determine the pressure perturbation, and finally, knowing $p$ and the shape of the surface elevation $\eta$, use (9) to determine the drag force.

This calculation is facilitated if all perturbed variables $(w, p$, and $\eta)$ are expressed as Fourier integrals along $x$ and $y$ :

$$
\begin{aligned}
& w(x, y, z)=\int_{-\infty}^{+\infty} \int_{-\infty}^{+\infty} \hat{w}\left(k_{1}, k_{2}, z\right) e^{i\left(k_{1} x+k_{2} y\right)} d k_{1} d k_{2}, \\
& p(x, y, z)=\int_{-\infty}^{+\infty} \int_{-\infty}^{+\infty} \hat{p}\left(k_{1}, k_{2}, z\right) e^{i\left(k_{1} x+k_{2} y\right)} d k_{1} d k_{2},
\end{aligned}
$$

$$
\eta(x, y)=\int_{-\infty}^{+\infty} \int_{-\infty}^{+\infty} \hat{\eta}\left(k_{1}, k_{2}\right) e^{i\left(k_{1} x+k_{2} y\right)} d k_{1} d k_{2} .
$$

Here, the Fourier transforms are denoted by a hat, and $k_{1}$ and $k_{2}$ are, respectively, the components of the wavenumber along $x$ and $y$.

Using (10), the equation for the vertical velocity perturbation (6) takes the form

$\hat{w}^{\prime \prime}+\left[\frac{N^{2} k_{12}^{2}}{\left(U k_{1}+V k_{2}\right)^{2}}-k_{12}^{2}-\frac{U^{\prime \prime} k_{1}+V^{\prime \prime} k_{2}}{U k_{1}+V k_{2}}\right] \hat{w}=0$,

where $k_{12}=\left(k_{1}^{2}+k_{2}^{2}\right)^{1 / 2}$ and the prime denotes differentiation with respect to $z$. This is sometimes called the Taylor-Goldstein equation, and is subject to the boundary condition

$$
\hat{w}(z=0)=i \hat{\eta}\left[U(z=0) k_{1}+V(z=0) k_{2}\right],
$$

which results from (7) and (10)-(12), and also to the radiation boundary condition at $z \rightarrow+\infty$. Similarly, using (10)-(11), the equation for the pressure (8) becomes

$$
\hat{p}=i \frac{\rho_{0}}{k_{12}^{2}}\left[\left(U^{\prime} k_{1}+V^{\prime} k_{2}\right) \hat{w}-\left(U k_{1}+V k_{2}\right) \hat{w}^{\prime}\right] .
$$

Finally, the equation for the drag (9) can be expressed as

$$
\mathbf{D}=4 \pi^{2} i \int_{-\infty}^{+\infty} \int_{-\infty}^{+\infty} \mathbf{k} \hat{p}^{*}(z=0) \hat{\eta} d k_{1} d k_{2},
$$

where $\mathbf{k}=\left(k_{1}, k_{2}, 0\right)$ is the horizontal wavenumber vector and the asterisk denotes complex conjugate.

Equation (13), although being linear with respect to $\hat{w}$, has a variable coefficient (between square brackets), and in general can only be solved by numerical or approximate analytical methods. Here, it will be solved using the WKB approximation, where it is assumed that the vertical structure of the solutions (and the background flow that determines them) varies slowly in the vertical. In order to use this approximation consistently, it is necessary first to introduce a scaled vertical coordinate $Z=\varepsilon z$, where $\varepsilon$ is a small parameter. The purpose of this change of variable is to bear out clearly the difference in magnitude of the various terms in the equation being treated. Whereas in the original equation [in this case (13)], the terms with vertical derivatives are presumably smaller than the others but are not clearly identified, in the equation with scaled vertical coordinate, to be presented next, the terms with vertical derivatives are of the same order of magnitude as the other terms, but multiplied by appropriate powers of $\varepsilon$, enabling a solution procedure in terms of power series. For example, when $z$ is replaced by $Z / \varepsilon$ in (13) and it is noted that $\partial / \partial z=\varepsilon \partial / \partial Z$, (13) becomes

$$
\varepsilon^{2} \ddot{\hat{w}}+\left[\frac{N^{2} k_{12}^{2}}{\left(U k_{1}+V k_{2}\right)^{2}}-k_{12}^{2}-\varepsilon^{2} \frac{\ddot{U} k_{1}+\ddot{V} k_{2}}{U k_{1}+V k_{2}}\right] \hat{w}=0,
$$


where differentiation with respect to $Z$ has been replaced by a dot, to simplify the notation.
The WKB solution to (17) (see, e.g., Bender and Orszag 1999) is

$$
\hat{w}(Z)=\hat{w}(Z=0) \exp \left\{i \varepsilon^{-1} \int_{0}^{Z}\left[m_{0}(\zeta)+\varepsilon m_{1}(\zeta)+\varepsilon^{2} m_{2}(\zeta)+\cdots\right] d \zeta\right\} .
$$

In this equation, the vertical wavenumber of the internal gravity waves $m(Z)$ has been expanded as a power series of $\varepsilon$, where the subscripts indicate the order of each term. This series is extended here up to second order because this is necessary for the vertical variation of the unperturbed velocity to have any impact on the surface drag, as will be shown.

When the solution (18) is introduced into (17), three separate equations result, valid at zeroth, first, and second order in $\varepsilon$. These equations, which determine the definitions of $m_{0}, m_{1}$, and $m_{2}$ are

$$
\begin{aligned}
-m_{0}^{2}+\frac{N^{2} k_{12}^{2}}{\left(U k_{1}+V k_{2}\right)^{2}}-k_{12}^{2} & =0, \\
i \dot{m}_{0}-2 m_{0} m_{1} & =0, \\
i \dot{m}_{1}-2 m_{0} m_{2}-m_{1}^{2}-\frac{\ddot{U} k_{1}+\ddot{V} k_{2}}{U k_{1}+V k_{2}} & =0 .
\end{aligned}
$$

As is easily noticed, when solved in succession, these three equations are algebraic for $m_{0}, m_{1}$, and $m_{2}$, yielding

$$
\begin{aligned}
m_{0}= & {\left[\frac{N^{2} k_{12}^{2}}{\left(U k_{1}+V k_{2}\right)^{2}}-k_{12}^{2}\right]^{1 / 2}, } \\
m_{1}= & -\frac{1}{2} i \frac{\dot{U} k_{1}+\dot{V} k_{2}}{U k_{1}+V k_{2}}\left[1-\frac{\left(U k_{1}+V k\right)^{2}}{N^{2}}\right]^{-1}, \\
m_{2}= & -\frac{1}{8} \frac{U k_{1}+V k_{2}}{N k_{12}} \\
& \times\left\{\left(\frac{\dot{U} k_{1}+\dot{V} k_{2}}{U k_{1}+V k_{2}}\right)^{2}\left[1-6 \frac{\left(U k_{1}+V k\right)^{2}}{N^{2}}\right]\right. \\
& \left.\times\left[1-\frac{\left(U k_{1}+V k_{2}\right)^{2}}{N^{2}}\right]^{-5 / 2}\right] \\
& +2 \frac{\ddot{U} k_{1}+\ddot{V}_{2}}{U k_{1}+V k_{2}}\left[1-2 \frac{\left(U k_{1}+V k_{2}\right)^{2}}{N^{2}}\right] \\
& \left.\times\left[1-\frac{\left(U k_{1}+V k_{2}\right)^{2}}{N^{2}}\right]^{-3 / 2}\right\} .
\end{aligned}
$$

It is worth noting at this point that $m_{1}$ can easily be expressed in terms of $m_{0}$, through $m_{1}=(1 / 2) i \dot{m}_{0} / m_{0}$ [this results from (20)]. When introduced in the solution (18) (ignoring for the moment $m_{2}$ ), this yields

$$
\hat{w}(z)=\hat{w}(z=0)\left[\frac{m_{0}(z=0)}{m_{0}(z)}\right]^{1 / 2} \exp \left[i \int_{0}^{z} m_{0}(\zeta) d \zeta\right]
$$

which is equivalent to the WKB solution proposed by Grisogono (1994) — his Eq. (2.4) — and also apparently equal to the solution used by Shutts (1995) and Shutts and Gadian (1999), although they do not present the expression explicitly [an explicit solution, albeit for the particular case of a linear wind profile, is given by Shutts (1998)]. However, Grisogono (1994) defines $m_{0}$ inconsistently, mistakenly equating it to the whole coefficient between brackets multiplying the second term of (13). In fact, when correctly scaled, the terms including the second derivatives of $(U, V)$ only appear at second order, as shown by (17) and (24). This is the primary cause why the drag expression of Grisogono (1994) contains a correction due to the curvature of the wind profile, when in fact that correction should not appear in a consistent treatment using the first-order solution (25).

As seen in (22), the definition of $m_{0}$ is exactly equal to that valid for an atmosphere with a constant wind. This simple definition enables one to estimate the conditions of validity of the WKB approximation and to define $\varepsilon$ more precisely. The WKB approximation is valid when the vertical wavenumber of the internal gravity waves varies over a distance that is much larger than the inverse of its value. Mathematically, this is equivalent to

$$
\frac{m^{\prime}(z)}{m^{2}(z)} \ll 1 .
$$

In order for the WKB approach to be sound, it is also necessary that the power series for the wavenumber in the exponent of (18) be asymptotic, that is, $\varepsilon \ll 1$. As long as this condition is satisfied, $m(z) \approx m_{0}(z)$, so a reasonable definition for $\varepsilon$ is

$$
\varepsilon=\frac{m^{\prime}}{m^{2}} \approx \frac{m_{0}^{\prime}}{m_{0}^{2}} \approx \frac{U^{\prime} k_{1}+V^{\prime} k_{2}}{N k_{12}} .
$$

In the last equality of (27), the hydrostatic approximation has been used, which corresponds to neglecting the $k_{12}^{2}$ term in the definition of $m_{0}(22)$. It is fairly obvious that $\left(U^{\prime} k_{1}+V^{\prime} k_{2}\right) /\left(N k_{12}\right)$ is of the same order of magnitude as $\left(U^{\prime 2}+V^{\prime 2}\right)^{1 / 2} / N$, which means that $\varepsilon$ $=O\left(\mathrm{Ri}^{-1 / 2}\right)$, where $\mathrm{Ri}=N^{2} /\left(U^{\prime 2}+V^{\prime 2}\right)$ is the Rich- 
ardson number of the flow. The small parameter of this model $\varepsilon$ is therefore inversely proportional to the square root of the Richardson number, and clearly the model is only formally valid for high $\mathrm{Ri}$. In fact, it will be seen later that the model is reasonably accurate even when $\mathrm{Ri}$ is of order one.

The WKB solution for $\hat{w}$ is completely defined by (18), together with (14) and (22)-(24). It is now necessary to calculate the associated pressure perturbation $\hat{p}$. In terms of the new vertical coordinate $Z$, the equation for the pressure (15) becomes

$$
\hat{p}=i \varepsilon \frac{\rho_{0}}{k_{12}^{2}}\left[\left(\dot{U} k_{1}+\dot{V} k_{2}\right) \hat{w}-\left(U k_{1}+V k_{2}\right) \dot{\hat{w}}\right] .
$$

Now, differentiating (18) with respect to $Z$ yields

$$
\dot{\hat{w}}=i \varepsilon^{-1}\left(m_{0}+\varepsilon m_{1}+\varepsilon^{2} m_{2}\right) \hat{w},
$$

so, on introducing (29) into (28), the pressure perturbation is given by

$$
\begin{aligned}
\hat{p}=i \frac{\rho_{0}}{k_{12}^{2}} & {\left[\varepsilon\left(\dot{U} k_{1}+\dot{V} k_{2}\right)\right.} \\
& \left.-i\left(U k_{1}+V k_{2}\right)\left(m_{0}+\varepsilon m_{1}+\varepsilon^{2} m_{2}\right)\right] \hat{w} .
\end{aligned}
$$

For the purpose of calculating the surface wave drag, only the pressure at the surface is required. Using the boundary condition at the surface (14) and (30), this can be written

$$
\begin{aligned}
& \hat{p}(z=0) \\
&=-\frac{\rho_{0}}{k_{12}^{2}}\{ \varepsilon\left(\dot{U}_{0} k_{1}+\dot{V}_{0} k_{2}\right)-i\left(U_{0} k_{1}+V_{0} k_{2}\right) \\
& \times\left[m_{0}(z=0)+\varepsilon m_{1}(z=0)\right. \\
&\left.\left.\quad+\varepsilon^{2} m_{2}(z=0)\right]\right\}\left(U_{0} k_{1}+V_{0} k_{2}\right) \hat{\eta},
\end{aligned}
$$

where the subscript 0 denotes values of the velocity or its derivatives taken at $z=0$. From (31), some conclusions can already be drawn about the surface drag. The drag results from the asymmetry of the pressure perturbation relative to the mountain, therefore a pressure perturbation that is proportional to the orography by a real factor does not contribute to the drag because it is in phase with the surface elevation. This implies that the first term within the square brackets in (31) does not contribute to the drag. Additionally, (23) reveals that $m_{1}$ is always pure imaginary, so the term involving $m_{1}$ is (31) does not contribute to the drag either. As a consequence, the only term in (31) that makes the surface drag for a flow with variable velocity differ from that associated with flow with constant velocity is that involving $m_{2}$. This explains why first-order WKB treatments, such as those of Shutts and Gadian (1999), fail to find any difference between the surface drag in the case of a slowly varying wind profile and a constant wind profile [see their Eq. (20)].

It is clear from (31) that, like the vertical wavenumber, the surface pressure is given by a power series of $\varepsilon$, namely,

$$
\hat{p}(z=0)=\hat{p}_{0}+\hat{p}_{1} \varepsilon+\hat{p}_{2} \varepsilon^{2}+\ldots,
$$

where

$\hat{p}_{0}=i \frac{\rho_{0}}{k_{12}^{2}}\left(U_{0} k_{1}+V_{0} k_{2}\right)^{2} m_{0}(z=0) \hat{\eta}$,

$\hat{p}_{1}=-\frac{\rho_{0}}{k_{12}^{2}}\left[\left(\dot{U}_{0} k_{1}+\dot{V}_{0} k_{2}\right)-i\left(U_{0} k_{1}+V_{0} k_{2}\right) m_{1}(z=0)\right]$

$$
\times\left(U_{0} k_{1}+V_{0} k_{2}\right) \hat{\eta}
$$

$\hat{p}_{2}=i \frac{\rho_{0}}{k_{12}^{2}}\left(U_{0} k_{1}+V_{0} k_{2}\right)^{2} m_{2}(z=0) \hat{\eta}$.

When (22)-(24) are used to substitute the definitions of $m_{0}, m_{1}$, and $m_{2}$ evaluated at $z=0,(33)-(35)$ take the form

$$
\begin{aligned}
\hat{p}_{0}= & i \frac{\rho_{0} N}{k_{12}}\left(U_{0} k_{1}+V_{0} k_{2}\right)\left[1-\frac{\left(U_{0} k_{1}+V_{0} k_{2}\right)^{2}}{N^{2}}\right]^{1 / 2} \hat{\eta} \\
\hat{p}_{1}= & -\frac{\rho_{0}}{k_{12}^{2}}\left(U_{0} k_{1}+V_{0} k_{2}\right)\left(\dot{U}_{0} k_{1}+\dot{V}_{0} k_{2}\right) \\
& \times\left\{1-\frac{1}{2}\left[1-\frac{\left(U_{0} k_{1}+V_{0} k_{2}\right)^{2}}{N^{2}}\right]^{-1}\right\} \hat{\eta}, \\
\hat{p}_{2}= & -\frac{1}{8} i \frac{\rho_{0}}{k_{12}^{3}} \frac{\left(U_{0} k_{1}+V_{0} k_{2}\right)^{3}}{N} \\
& \times\left\{\left(\frac{\left.\dot{U}_{0} k_{1}+\dot{V}_{0} k_{2}\right)^{2}\left[1-6 \frac{\left(U_{0} k_{1}+V_{0} k_{2}\right)^{2}}{U_{0} k_{1}+V_{0} k_{2}}\right)^{2}}{N^{2}}\right]^{-5 / 2}\right. \\
& \left.\times\left[1-\frac{\left(U_{0} k_{1}+V_{0} k_{2}\right)^{2}}{N^{2}}\right]^{-3 / 2}\right\} \hat{\eta} . \\
& +2 \frac{\ddot{U}_{0} k_{1}+\ddot{V}_{0} k_{2}}{U_{0} k_{1}+V_{0} k_{2}}\left[1-2 \frac{\left(U_{0} k_{1}+V_{0} k_{2}\right)^{2}}{N^{2}}\right] \\
& \left.\times\left[1-\frac{\left(U_{0} k_{1}+V_{0} k_{2}\right)^{2}}{N^{2}}\right]^{-3 / 2}\right]
\end{aligned}
$$

These expressions are somewhat lengthy, but simplify considerably when the hydrostatic approximation is assumed, as will be seen next.

\section{Hydrostatic flow}

The hydrostatic approximation is acceptable in a wide range of situations of practical interest, and will be adopted here to simplify the calculations, since only then can an expression for the wave drag be derived analytically. Given the dimensionless width of the mountain $\hat{a}=N a /|\mathbf{U}|$ (where $a$ is the corresponding dimensional half-width), the hydrostatic approximation is formally valid when $\hat{a} \gg 1$. Since, in the WKB approximation, the unperturbed flow is assumed to vary slowly, this 
dimensionless quantity can be defined alternativley in terms of the surface velocity $\hat{a}=N a /\left|\mathbf{U}_{0}\right|$. When this parameter tends to infinity, all second terms within the square brackets in the expressions for $\hat{p}_{0}, \hat{p}_{1}$, and $\hat{p}_{2}$, (36)-(38), vanish, because they are clearly proportional to $\hat{a}^{-2}$. Hence, the equations giving the pressure perturbations at the surface become

$$
\begin{aligned}
\hat{p}_{0}=i \rho_{0} \frac{N}{k_{12}}\left(U_{0} k_{1}+V_{0} k_{2}\right) \hat{\eta} \\
\hat{p}_{1}=-\frac{1}{2} \frac{\rho_{0}}{k_{12}^{2}}\left(U_{0} k_{1}+V_{0} k_{2}\right)\left(\dot{U}_{0} k_{1}+\dot{V}_{0} k_{2}\right) \hat{\eta} \\
\hat{p}_{2}=-\frac{1}{8} i \frac{\rho_{0}}{N k_{12}^{3}}\left[\left(U_{0} k_{1}+V_{0} k_{2}\right)\left(\dot{U}_{0} k_{1}+\dot{V}_{0} k_{2}\right)^{2}\right. \\
\left.\quad+2\left(\ddot{U}_{0} k_{1}+\ddot{V}_{0} k_{2}\right)\left(U_{0} k_{1}+V_{0} k_{2}\right)^{2}\right] \hat{\eta}
\end{aligned}
$$

From (16) and (32), it follows that the drag force can also be expressed as a power series of $\varepsilon$ :

$$
\mathbf{D}=\mathbf{D}_{0}+\mathbf{D}_{1} \varepsilon+\mathbf{D}_{2} \varepsilon^{2}+\ldots,
$$

where

$$
\begin{gathered}
\mathbf{D}_{j}=4 \pi^{2} i \int_{-\infty}^{+\infty} \int_{-\infty}^{+\infty} \mathbf{k} \hat{p}_{j}^{*} \hat{\eta} d k_{1} d k_{2} \\
(j=0,1,2, \ldots) .
\end{gathered}
$$

Before $\mathbf{D}_{0}, \mathbf{D}_{1}$, and $\mathbf{D}_{2}$ can be calculated, however, the shape of the orography must be specified. In this study, following Smith (1980), Grubišić and Smolarkiewicz (1997), and Shutts and Gadian (1999), a circular bellshaped isolated mountain will be adopted. This type of orography is defined by

$$
\eta(x, y)=\frac{h_{0}}{\left[1+(x / a)^{2}+(y / a)^{2}\right]^{3 / 2}},
$$

and the corresponding Fourier transform, which is required in (43) and (39)-(41), is

$$
\hat{\eta}\left(k_{1}, k_{2}\right)=\frac{1}{2 \pi} h_{0} a^{2} e^{-a k_{12}} .
$$

\section{Results}

If the expressions for the pressure presented in the previous section, (39)-(41), are substituted in the corresponding equations for the drag, if (45) is used and the integration over all wavenumbers is carried out analytically (by adopting polar coordinates), one obtains

$$
\begin{aligned}
& \mathbf{D}_{0}=\frac{\pi}{4} \rho_{0} N \mathbf{U}_{0} a h_{0}^{2}, \\
& \mathbf{D}_{1}=0
\end{aligned}
$$

$$
\begin{aligned}
D_{2 x}= & -\frac{\pi}{4} \rho_{0} N U_{0} a h_{0}^{2} \\
\times & {\left[\frac{1}{32}\left(3 \frac{\dot{U}_{0}^{2}}{N^{2}}+\frac{\dot{V}_{0}^{2}}{N^{2}}+2 \frac{V_{0}}{U_{0}} \frac{\dot{U}_{0} \dot{V}_{0}}{N^{2}}\right)\right.} \\
& \left.+\frac{1}{16}\left(3 \frac{\ddot{U}_{0} U_{0}}{N^{2}}+\frac{V_{0}}{U_{0}} \frac{\ddot{U}_{0} V_{0}}{N^{2}}+2 \frac{\ddot{V}_{0} V_{0}}{N^{2}}\right)\right], \\
D_{2 y}=- & \frac{\pi}{4} \rho_{0} N V_{0} a h_{0}^{2} \\
\times & {\left[\frac{1}{32}\left(3 \frac{\dot{V}_{0}^{2}}{N^{2}}+\frac{\dot{U}_{0}^{2}}{N^{2}}+2 \frac{U_{0}}{V_{0}} \frac{\dot{U}_{0}}{N^{2}}\right)\right.} \\
& \left.+\frac{1}{16}\left(3 \frac{\ddot{V}_{0} V_{0}}{N^{2}}+\frac{U_{0}}{V_{0}} \frac{\ddot{V}_{0}}{N_{0}^{2}}+2 \frac{\ddot{U}_{0} U_{0}}{N^{2}}\right)\right]
\end{aligned}
$$

From these expressions, it can immediately be confirmed that there is no contribution to the drag at first order in $\varepsilon$. This was to be expected from (40), because $\hat{p}_{1}$ has no imaginary part. As expected, the zeroth-order $\operatorname{drag} \mathbf{D}_{0}=\left(D_{0 x}, D_{0 y}\right)$ is equal to that derived by Smith (1980) for a constant wind profile. The second-order drag is nonzero and has a complicated form, depending on the first and second derivatives of the unperturbed velocity profile. A detailed interpretation of the contributions of the zeroth-, first-, and second-order parts of the pressure perturbation to the drag will be given later.

When using polar coordinates to integrate (43), it becomes clear that $\hat{p}_{0}, \hat{p}_{1}$, and $\hat{p}_{2}$ do not depend explicitly on the value of the wavenumber $k_{12}$, but only on the azimuthal angle [see (39)-(41)], so the integration over $k_{12}$ is equal for $\mathbf{D}_{0}, \mathbf{D}_{1}$, and $\mathbf{D}_{2}$ (i.e., amounts to multiplication by the same constant, determined by the shape of the orography). This means that, although the absolute values of $\mathbf{D}_{0}, \mathbf{D}_{1}$, and $\mathbf{D}_{2}$ depend on the shape of the orography, their relative magnitude does not, as long as the orography is axisymmetric. This property adds much relevance to the present calculations, which therefore are applicable to any axisymmetric mountain. In nonhydrostatic conditions, this property does not hold, because nonhydrostatic effects depend on the value of the wavenumber, as is evident by looking at the second terms between square brackets in (36)-(38), and this dependence is different for $\hat{p}_{0}, \hat{p}_{1}$, and $\hat{p}_{2}$. This property does not hold either for nonaxisymmetric orography, because in that case $\hat{\eta}$ depends on the azimuthal angle and this modifies the integration over this variable in (43).

Adding the three terms of (42) in the form given by (46) $-(49)$ and noting that $(\cdots)^{\prime}=\varepsilon(\cdot \cdot)$ yields

$$
\begin{aligned}
D_{x}=D_{0 x} & {\left[1-\frac{1}{32}\left(3 \frac{U_{0}^{\prime 2}}{N^{2}}+\frac{V_{0}^{\prime 2}}{N^{2}}+2 \frac{V_{0}}{U_{0}} \frac{U_{0}^{\prime} V_{0}^{\prime}}{N^{2}}\right)\right.} \\
& \left.-\frac{1}{16}\left(3 \frac{U_{0}^{\prime \prime} U_{0}}{N^{2}}+\frac{V_{0}}{U_{0}} \frac{U_{0}^{\prime \prime} V_{0}}{N^{2}}+2 \frac{V_{0}^{\prime \prime} V_{0}}{N^{2}}\right)\right],
\end{aligned}
$$




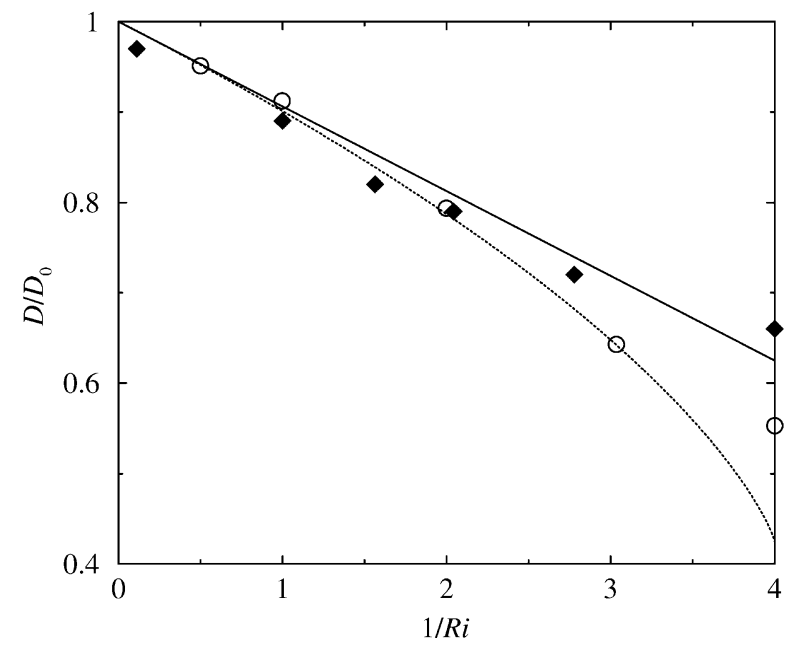

FIG. 1. Normalized drag as a function of the inverse Richardson number for the flow given by (52). (solid line) Present model (53); (dotted line) analytical model of Grubišić and Smolarkiewicz (1997); (diamonds) numerical simulations by Grubišić and Smolarkiewicz (1997; $\hat{a}=5, \hat{h} \leq 0.1)$; and (circles) numerical simulations using the NH3D model $(\hat{a}=23, \hat{h}=0.01)$.

$$
\begin{aligned}
D_{y}=D_{0 y}[ & -\frac{1}{32}\left(3 \frac{V_{0}^{\prime 2}}{N^{2}}+\frac{U_{0}^{\prime 2}}{N^{2}}+2 \frac{U_{0}}{V_{0}} \frac{U_{0}^{\prime} V_{0}^{\prime}}{N^{2}}\right) \\
& \left.-\frac{1}{16}\left(3 \frac{V_{0}^{\prime \prime} V_{0}}{N^{2}}+\frac{U_{0}}{V_{0}} \frac{V_{0}^{\prime \prime} U_{0}}{N^{2}}+2 \frac{U_{0}^{\prime \prime} U_{0}}{N^{2}}\right)\right]
\end{aligned}
$$

for the $x$ and $y$ components of the drag. These expressions, which are valid for any axisymmetric orography, are the main result of this study.

The mathematical form of (50)-(51) remains too complicated and general to be readily understood. For that reason, three simplified flows will be considered next to test the accuracy of these equations against numerical simulation results. These results are taken from Grubišić and Smolarkiewicz (1997) and also calculated using the nonhydrostatic mesoscale model NH3D, developed by Miranda (see Miranda and James 1992).

Since the comparisons will focus on the dependence of the drag on the variation of the mean velocity profile, and this is quantified by the Richardson number, flows with a Richardson number that is constant in height will be addressed for simplicity.

\section{a. Unidirectional wind shear: Velocity with a linear variation}

Arguably the simplest flow with a constant Richardson number is that where the wind vector has only one component and that component varies linearly with height:

$$
U=U_{0}+\alpha z, \quad V=0,
$$

where $\alpha$ is a constant. This was the velocity profile considered by Smith (1986) for flow over a ridge and by Grubišić and Smolarkiewicz (1997) for flow over an isolated mountain. In this case, the Richardson number is given by $\mathrm{Ri}=N^{2} / U_{0}^{\prime 2}=N^{2} / \alpha^{2}$. The expressions obtained with the present model for the surface drag result directly from (50)-(51) when $U_{0}^{\prime \prime}=V_{0}=V_{0}^{\prime}=$ $V_{0}^{\prime \prime}=0$, and are

$$
D_{x}=D_{0 x}\left(1-\frac{3}{32 \mathrm{Ri}}\right), \quad D_{y}=0
$$

where $D_{0 x}$ is the drag for a constant wind of magnitude $\left(U_{0}, V_{0}\right)$ (i.e., in the absence of shear). So, the drag has the same direction as the unperturbed flow at the surface, but its magnitude decreases as the shear increases (or alternatively as the Richardson number decreases). This type of qualitative behavior has been predicted by the exact analytical treatment of Grubišić and Smolarkiewicz (1997), but the dependence on the Richardson number was not so simple as here, involving elliptic integrals. It can, however, be shown that the analytical drag expression Grubišić and Smolarkiewicz [1997, their Eq. (28)] tends asymptotically to (53) for high $\mathrm{Ri}$, when only the term that depends linearly on $\mathrm{Ri}^{-1}$ is retained in the corresponding expansion. This is a clear indication that the present model is sound.

Figure 1 shows the drag force $D=D_{x}$ normalized by the drag in the absence of shear $D_{0}=D_{0 x}$ as a function the inverse Richardson number $\mathrm{Ri}^{-1}$. Since in (53) the departure of the drag from the unsheared case is inversely proportional to $\mathrm{Ri}$, the corresponding curve is a straight line. Numerical simulation data obtained in approximately linear and hydrostatic conditions by Grubišić and Smolarkiewicz (1997), and results from the exact formula derived by the same authors, which represents finite shear rates exactly, are also displayed as the diamonds and the dahsed curve, respectively. Numerical simulation data from the NH3D numerical model are shown as the circles. It can be seen that the present analytical model reproduces quite well the behavior of the numerical data, even better than the exact theory for relatively low $\mathrm{Ri}$ in the case of Grubišić and Smolarkiewicz's data, which is surprising. This is fortuitous and presumably due to factors that are not taken into account by either analytical models. One possibility is that $\hat{a}$ is too small in the numerical simulations of Grubišić and Smolarkiewicz (1997). This is apparently confirmed by the fact that their exact formula is in better agreement than (53) with data from the NH3D model, where conditions are more nearly hydrostatic. However, results are qualitatively similar and, due to its simplicity, (53) seems more appropriate for guiding estimates of the effect of shear on the drag.

Figure 2 displays horizontal cross sections of the pres- 

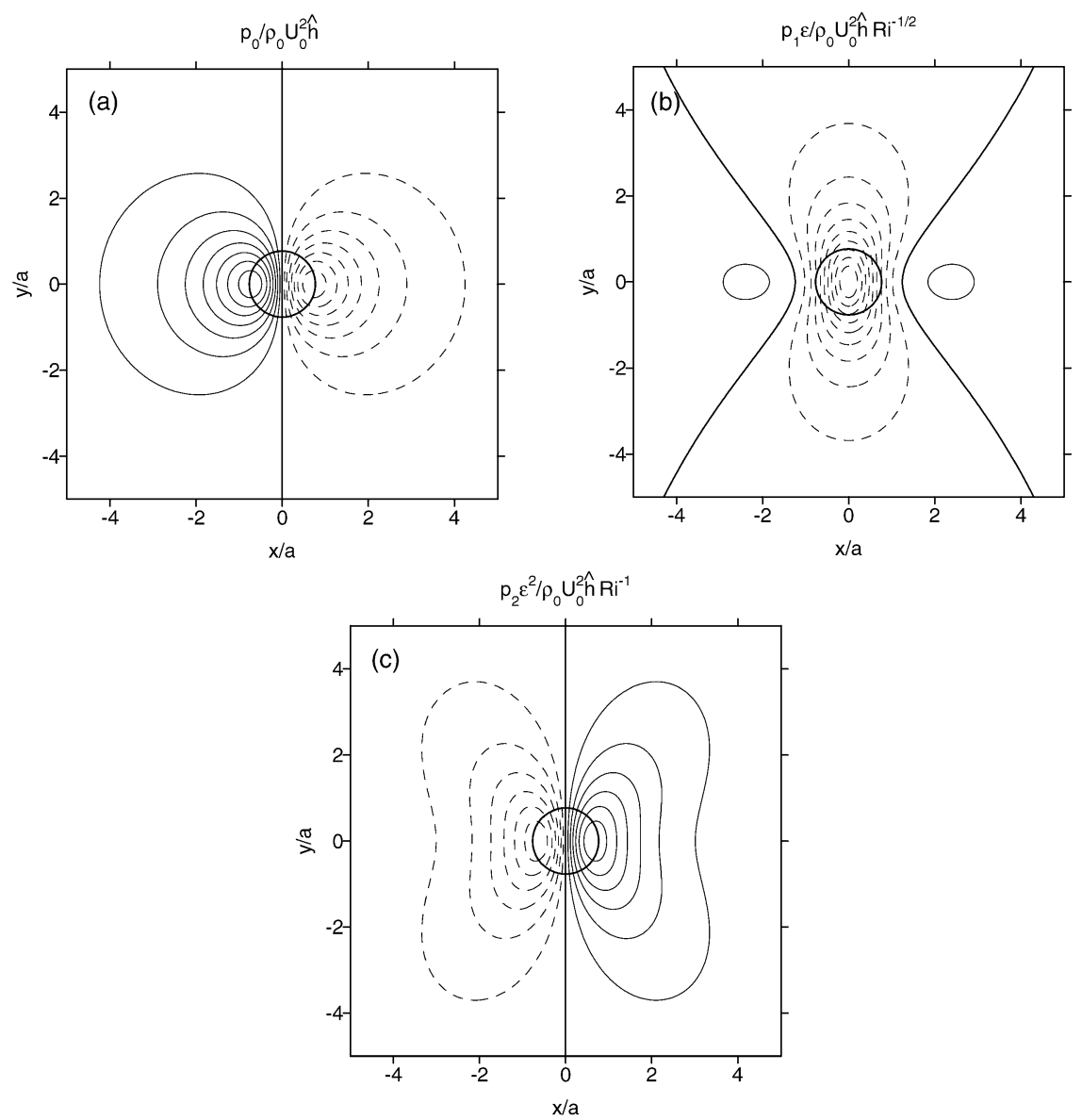

FIG. 2. Cross sections of the contributions to the normalized pressure perturbation at the surface given by the present model for the flow (52). (thick solid line) Terrain elevation equal to $0.5 h_{0}$; (solid contours) positive values; (dashed contours) negative values. (a) Zeroth-order pressure, contour spacing 0.05; (b) first-order pressure, contour spacing 0.025; and (c) second-order pressure, contour spacing 0.005 .

sure perturbation at the surface calculated with the present model. The zeroth-, first-, and second-order contributions to the pressure field are separated to emphasize their different roles. This has been done using the expressions (39)-(41), which were inversely transformed back to physical space using a fast Fourier transform (FFT) algorithm (Press et al. 1992). Figure 2a shows the zeroth-order pressure perturbation, which is antisymmetric relative to the mountain, having a positive maximum on the upwind side and a negative minimum on the downwind side (cf. Fig. 3 of Smith 1980). This causes a positive drag on the mountain, as is well known. The first-order pressure perturbation, on the other hand (Fig. 2b), is symmetric, having a negative minimum, elongated in the spanwise direction, exactly above the mountain top and therefore producing no drag. It is for this reason there is no term proportional to $\mathrm{Ri}^{-1 / 2}$ in the expression for the drag (53). The secondorder pressure perturbation (Fig. 2c) is antisymmetric like $p_{0}$, but has the opposite sign, with a negative minimum on the upwind side of the mountain and a positive maximum on the downwind side. The effect of the $p_{2}$ field (which is also somewhat elongated in the spanwise direction), is therefore to oppose $p_{0}$, making the drag decrease. This is of course consistent with the negative term proportional to $\mathrm{Ri}^{-1}$ in (53).

Figure 3 compares the sum of these three pressure contributions, for a flow given by (52) where $\alpha<0$ (negative shear) and $\mathrm{Ri}=0.5$, with a similar flow computed by Grubišić and Smolarkiewicz (1997) using their nonlinear numerical model. The total pressure perturbation shown in Fig. 3a is wedge shaped, with the pressure maximum shifted toward the mountain top. Note how these qualitative features are in close agreement with those quoted by Grubišić and Smolarkiewicz (1997) and visible in Fig. 3b. Figure 3a is also remarkably similar to Fig. $6 \mathrm{~b}$ of Grubišić and Smolarkiewicz (1997), where the same field is computed using their 

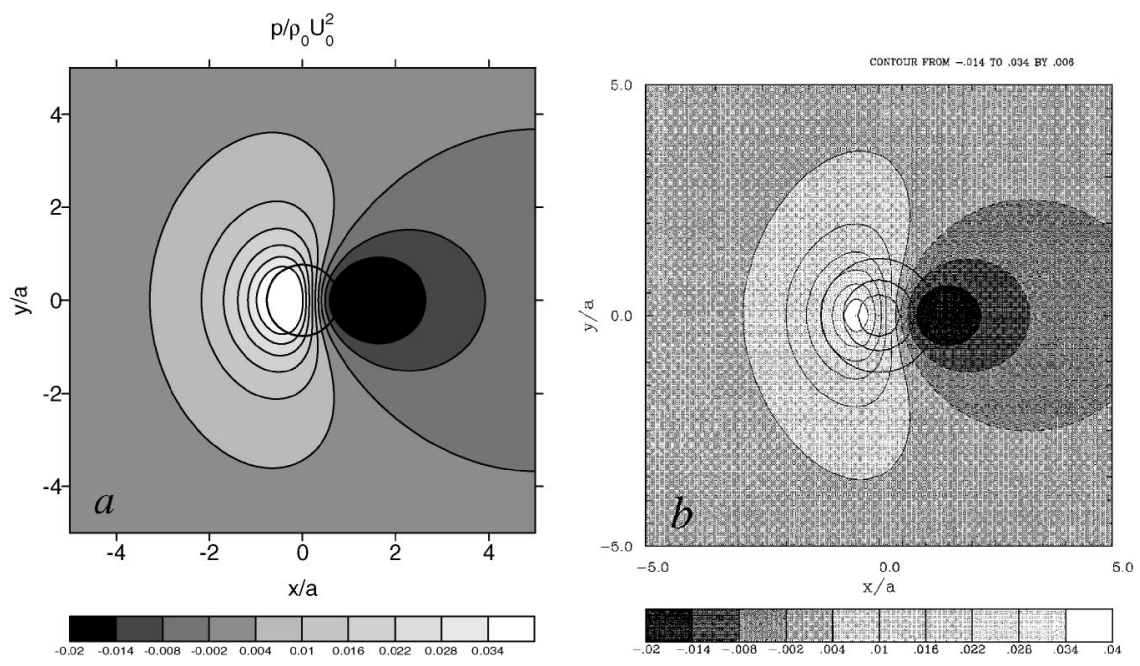

FIG. 3. Normalized total pressure perturbation at the surface for the flow given by (52) when $\mathrm{Ri}=0.5$. Shading similar to that used in Figs. 6 and 10 of Grubišić and Smolarkiewicz (1997). (a) Present model, (thick solid line) terrain elevation equal to $0.5 h_{0}$; and (b) numerical simulations of Grubišić and Smolarkiewicz (1997; reproduction of their Fig. 10b).

exact analytical model. This confirms what was already suggested by Fig. 1: that the present model gives accurate and useful results for $\mathrm{Ri}=O(1)$.

\section{b. Directional wind shear: Velocity with a linear variation}

One of the simplest flows that changes direction with height is that where one of the velocity components varies linearly and the other is constant:

$$
U=U_{0}+\alpha z, \quad V=V_{0} .
$$

With $U_{0}=0$, this was the type of wind profile used in the studies of Shutts (1995) and Shutts and Gadian (1999), because it also enables an analytical resolution of the equations of motion for arbitrary shear rates. In this section it will be assumed instead that $V_{0}=U_{0} \neq$ 0 . This velocity profile corresponds to a constant Richardson number, given, as in the previous case, by $\mathrm{Ri}=$ $N^{2} / U_{0}^{\prime 2}=N^{2} / \alpha^{2}$.

There are, however, important differences in the way the expressions for the drag, (50)-(51), simplify. Now, $U_{0}^{\prime \prime}=V_{0}^{\prime}=V_{0}^{\prime \prime}=0$, and this yields

$$
D_{x}=D_{0 x}\left(1-\frac{3}{32 \mathrm{Ri}}\right), \quad D_{y}=D_{0 y}\left(1-\frac{1}{32 \mathrm{Ri}}\right) \text {. }
$$

Hence, the drag in the $x$ direction (the direction along which there is shear) decreases as rapidly with the Richardson number as in the previous case, but the drag in the $y$ direction (along which the wind velocity is constant) decreases $1 / 3$ more slowly as Ri decreases. This leads to a phenomenon specific to the present type of flow: a misalignment of the drag force with the unperturbed velocity vector at the surface. This misalignment is greater for smaller Richardson numbers, as (55) im- plies. It was impossible to find in the literature numerical simulation data appropriate for comparing with (55). For example the simulations of Shutts (1995) and Shutts and Gadian (1999), which consider the same type of flow, are for very high Richardson numbers $(\mathrm{Ri} \approx 100)$. Equation (55) suggests that the corrections to the drag due to $\mathrm{Ri}$ are in that case insignificant. This result has been corroborated by Shutts and Gadian (1999) when they found good agreement between the numerical simulation data and their analytical expression for the drag (which ignores the effect of the Richardson number). So it was necessary to perform new numerical simulations for $\mathrm{Ri}=O(1)$ using the NH3D model (Miranda and James 1992). Again, approximately linear and hydrostatic conditions were considered in these simulations.

Figure 4 shows the drag expressions in (55), respectively, as the solid and dotted lines, against data from the NH3D numerical model, respectively, represented by the circles and the squares. It can be seen that, although (55) tends to overestimate the values of the data for $D_{x} / D_{0 x}$ at low Ri, the agreement is quite good, especially for high $\mathrm{Ri}$, consistent with the WKB approximation. The relative dependence of the two components of the drag on the Richardson number is particularly well captured, indicating that the angle between the drag force and the surface wind should be fairly well predicted.

Note that, in this situation, as well as in the situation treated in the previous section, the value taken by the drag does not depend, at second order in $\varepsilon$, on the sign of the shear (the Richardson number only depends on $U_{0}^{\prime}$ squared). So, a backward shear produces, to this degree of approximation, the same modification to the drag as forward shear (cf. Grubišić and Smolarkiwicz 


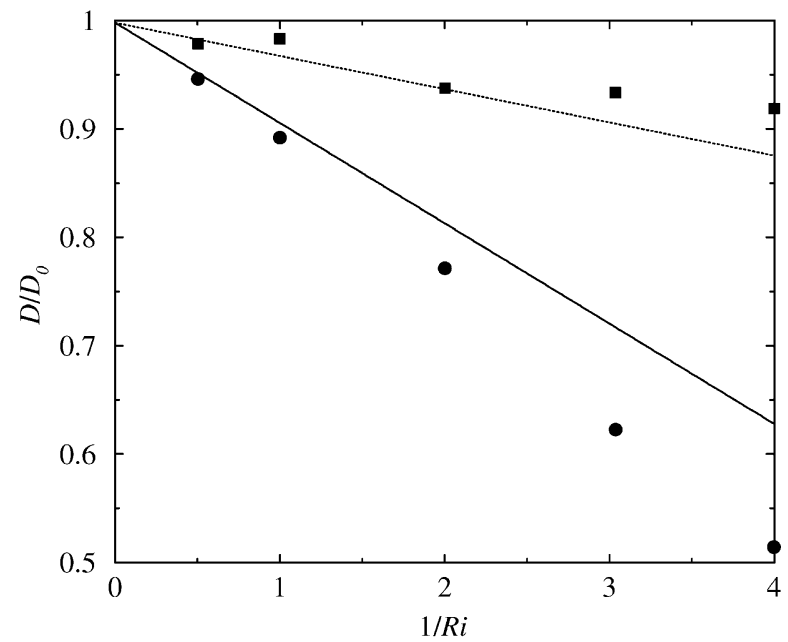

FIG. 4. Normalized drag as a function of the inverse Richardson number for the flow given by (54). (solid and dotted lines) The $D_{x}$ and $D_{y}$ from the present model (55); (circles and squares) $D_{x}$ and $D_{y}$ from numerical simulations using the NH3D model $(\hat{a}=23, \hat{h}=$ $0.01)$.

1997). Using the NH3D numerical model, it has been verified that, in practice, this is not so, and the drag in both directions decreases much more rapidly with $\mathrm{Ri}$ for forward shear than for backward shear. This may be a consequence of the fact that the hydrostatic approximation (assumption that $\hat{a}$ is large) is not as well satisfied by a flow with forward shear as by a flow with backward shear. Whereas in a flow with backward shear, the value of the velocity $\left(U^{2}+V^{2}\right)^{1 / 2}$ first decreases with height, rendering $\hat{a}$ progressively larger immediately above the surface (being even infinite at the critical level of a unidirectional flow), in a flow with forward shear the opposite occurs. It appears that, for Richardson numbers as low as those considered in Figs. 1 and 4, $\hat{a}$ immediately above the surface in the case of forward shear is so small that (55) can no longer be used. For this reason, in this and in the previous section, only backward shear cases have been considered. However, results not presented here, calculated with the NH3D model, show that, qualitatively, the behavior of the drag in the case of forward shear is the same, namely, the drag decreases as Ri decreases in the $x$ direction, and decreases more slowly in the $y$ direction.

Figure 5 shows the pressure perturbation for the present flow situation (54). While the $p_{0}$ field, presented in Fig. 5a, is exactly identical to that in Fig. 2a rotated by $45^{\circ}$ (due to the rotation of the surface wind by that angle), the $p_{1}$ and $p_{2}$ fields, presented in Figs. 5b and $5 c$, are qualitatively different from those of Figs. $2 b$ and $2 \mathrm{c}$. Over the mountain $p_{1}$ still has a negative minimum, therefore producing no drag, but is rotated relative to Fig. $2 \mathrm{~b}$ by an angle that is smaller than $45^{\circ}$. Field $p_{2}$ is rotated by an even smaller angle. There are also some differences in the magnitude of the first- and secondorder terms of the pressure perturbation, with $p_{1}$ and $p_{2}$ being somewhat smaller in Figs. 5b and 5c than in Figs. $2 \mathrm{~b}$ and $2 \mathrm{c}$. The important point in interpreting (55) is that the relatively small rotation of $p_{2}$ relative to Fig. $2 \mathrm{c}$ counteracts the $p_{0}$ pressure field more effectively along $x$ than along $y$. For that reason, the coefficient multiplying $\mathrm{Ri}^{-1}$ is larger in $D_{x}$ than in $D_{y}$. This is an interesting effect, which deserves more detailed investigation.

Figure 6 compares the total pressure perturbation, which is the sum of these three pressure contributions, with results from the NH3D model, for $\mathrm{Ri}=0.5$. Again, the total pressure field shown in Fig. 6a is wedge shaped, with the maximum slightly shifted toward the mountain top, and it is rotated from the $x$ direction due to the oblique orientation of the surface wind, but this rotation is by more than $45^{\circ}$. The pressure pattern is more asymmetric than in Fig. 3a, due to the different orientations of $p_{0}, p_{1}$, and $p_{2}$. This configuration compares very well with the pressure field computed using the NH3D numerical model, shown in Fig. 6 b.

\section{c. Directional wind shear: Velocity that turns with height}

Another type of flow, which has been used in idealized studies of mountain waves, is that where the wind rotates with height at a constant rate, while maintaining its magnitude (Shutts and Gadian 1999; Valente 2000). This flow can be represented as

$$
U=U_{0} \cos (\beta z), \quad V=U_{0} \sin (\beta z),
$$

where $\beta$ is a constant. By differentiating this expression, it can be seen that the Richardson number, which in general is defined by $\mathrm{Ri}=N^{2} /\left(U^{\prime 2}+V^{\prime 2}\right)$, is related to $\beta$ by $\mathrm{Ri}=N^{2} /\left(U_{0} \beta\right)^{2}$. It is therefore constant, as in the previous cases. For this particular type of flow, the dimensionless mountain width $\hat{a}=\mathrm{Na} /\left(U^{2}+V^{2}\right)^{1 / 2}$ takes the value $\hat{a}=N a / U_{0}$, which is also constant. This flow is characterized at the surface by $U_{0}^{\prime}=V_{0}=V_{0}^{\prime \prime}$ $=0$. Therefore, the Richardson number at the surface (which is equal in value to Ri at any height) can also be defined as Ri $=N^{2} / V_{0}^{\prime 2}$. Another aspect to be noted is that, unlike the previous flows, the present flow has a velocity vector with nonzero second and all other higher derivatives. This proves to be the key in determining the variation of the drag with Ri.

In order to obtain a simple expression for the drag in this case, it is convenient to relate the terms involving second derivatives of $U$ and $V$ in (50)-(51) to the Richardson number. This can be done by noting that, when (56) is valid, $U^{\prime \prime} U=-V^{\prime 2}$, and hence $U_{0}^{\prime \prime} U_{0}=-V_{0}^{\prime 2}$. Then it can be shown from (50)-(51) that the drag for the present flow is given by

$$
D_{x}=D_{0 x}\left(1+\frac{5}{32 \mathrm{Ri}}\right), \quad D_{0 y}=0 .
$$

Two interesting features immediately stand out from 

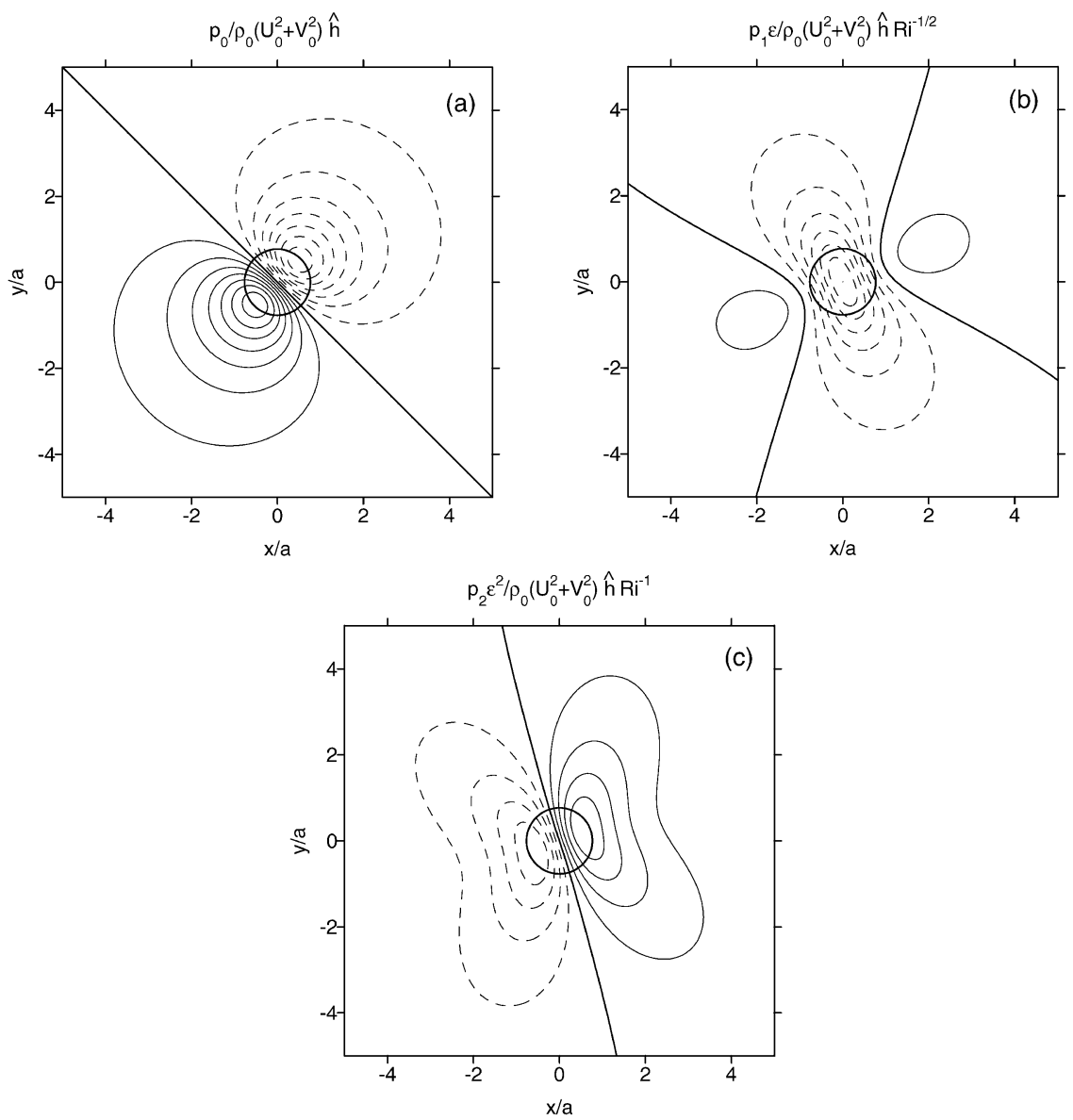

FIG. 5. Cross sections of the contributions to the normalized pressure perturbation at the surface given by the present model for the flow (54). (thick solid line) Terrain elevation equal to $0.5 h_{0}$; (solid contours) positive values; (dashed contours) negative values. (a) Zeroth-order pressure, contour spacing 0.05; (b) first-order pressure, contour spacing 0.025; and (c) second-order pressure, contour spacing 0.005 .
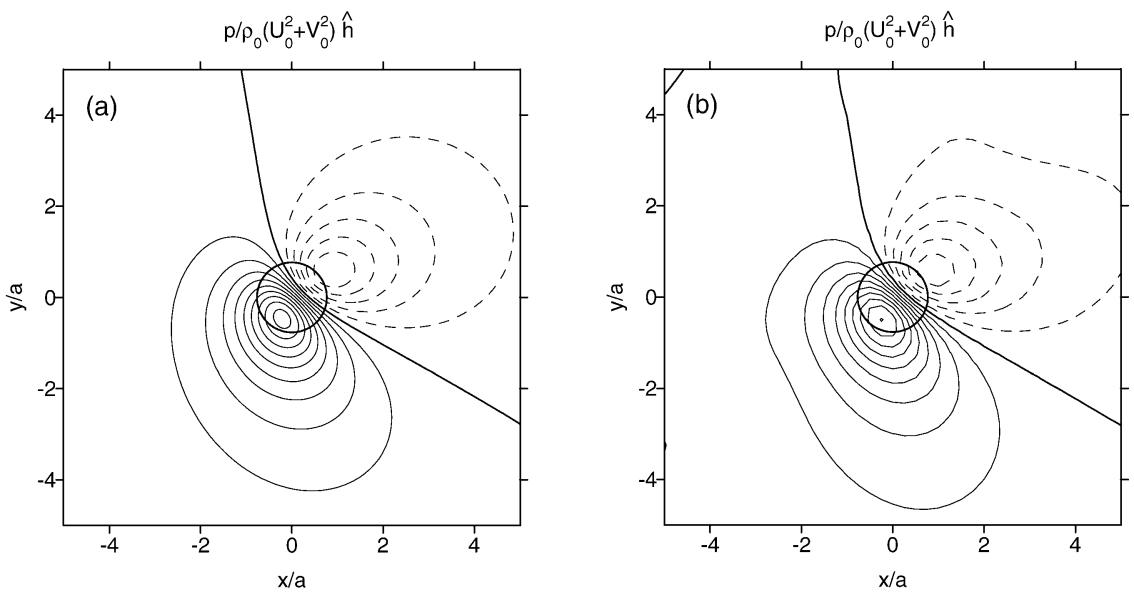

FIG. 6. Normalized total pressure perturbation at the surface for the flow given by (54) when $\mathrm{Ri}=0.5$. (thick solid line) Terrain elevation equal to $0.5 h_{0}$; (solid contours) positive values; (dashed contours) negative values, contour spacing 0.05. (a) Present model; and (b) NH3D numerical model. 


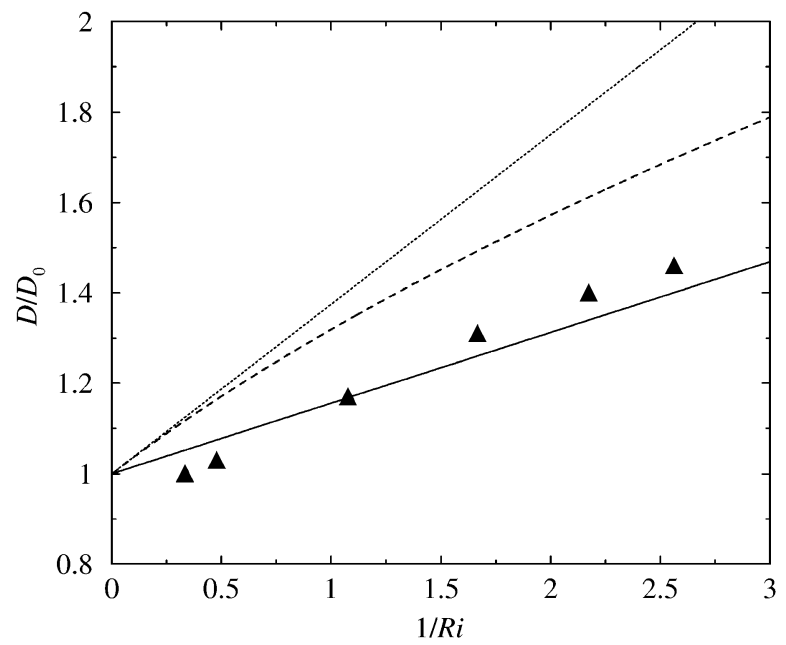

FIG. 7. Normalized drag as a function of the inverse Richardson number for the flow given by (56). (solid line) Present model (57); (dashed line) WKB model analogous to that of Grisogono (1994); (dotted line) corresponding first-order asymptotic expansion (58); (symbols) numerical simulations by Valente $(2000 ; \hat{a}=7.6, \hat{h}=0.08)$.

these expressions. First, there is no drag along the $y$ direction, although the flow rotates with height. In this sense, the drag behavior is different (and simpler) from that displayed in the previous section. Although a drag component along $y$ cannot be ruled out at higher order in $\varepsilon$, to second order the drag always takes the direction of the surface wind, which is along $x$. This result is supported by the nonlinear numerical simulations of Miranda (1991) and Valente (2000). Second, the drag increases as Ri decreases, which is a behavior opposite that displayed in the previous cases. This latter difference is due to the effect of the second derivative of the unperturbed velocity, that is, the curvature of the wind profile. The term involving $U_{0}^{\prime \prime}$, when negative (as is the case here), gives a positive contribution to $D_{x}$ in (50). Although this is counteracted by a small negative contribution from the term involving $V_{0}^{\prime 2}$, the dominant contribution at second order in $\varepsilon$, that is, proportional to $\mathrm{Ri}^{-1}$, remains positive.

Figure 7 shows the variation of the normalized drag $D / D_{0}=D_{x} / D_{0 x}$ with $\mathrm{Ri}^{-1}$. The solid line corresponds to (57) and the symbols to data from numerical simulations carried out by Valente (2000) using the NH3D numerical model (once more, in approximately linear and hydrostatic conditions). The dashed line corresponds to an expression for the drag derived using a WKB approach akin to that of Grisogono (1994), more precisely using the first-order solution (25) but including in $m_{0}$ the terms involving the curvature of the wind velocity profile. The dotted line is the corresponding first-order asymptotic expansion valid for high $\mathrm{Ri}$, which can be shown to have the form

$$
D_{x}=D_{0 x}\left(1+\frac{3}{8 \mathrm{Ri}}\right) .
$$

It can be noticed that (57) gives the best agreement with the numerical simulation data, while the curves based on Grisogono's approach overestimate the drag values, especially the asymptotic approximation (58). This failure is obviously due to the inconsistencies in the derivation of these expressions. Note in particular that the coefficient $3 / 8$ predicted by (58) is approximately twice the coefficient $5 / 32$ predicted by the correct WKB formula (57). If the flow was unidirectional $\left(V_{0}=V_{0}^{\prime}=\right.$ $V_{0}^{\prime \prime}=0$ ), the coefficient in (57) would be $3 / 16$, so this factor of 2 would be exact instead of approximate.

Figure 8 shows cross sections of the zeroth-, first-, and second-order contributions to the pressure perturbation; $p_{0}$ is identical in shape and intensity to that presented in section $3 \mathrm{a}$ (because the flow is aligned in the $x$ direction at the surface), but $p_{1}$ and $p_{2}$ are different; $p_{1}$ is weak and has positive maxima and negative minima placed, respectively, in the first and third quadrants, and in the second and fourth quadrants of the plot. The symmetry of these pressure patterns implies that there is again no drag at first order in $\varepsilon$, as (57) confirms. The $p_{2}$ field, on the other hand, is perfectly antisymmetric in the $x$ direction, justifying why there is no drag component along $y$. The pressure field is elongated in the $y$ direction, and tends to reinforce $p_{0}$, having a positive maximum on the upwind slope of the mountain and a negative minimum on the downwind slope. Besides having the opposite sign, the maximum and minimum in Fig. 8c are approximately 5/3 larger in magnitude than those in Fig. 2c, reflecting the difference in the coefficients in the corresponding drag formulas (respectively, $3 / 32$ and 5/32).

Figure $9 \mathrm{a}$ presents the total pressure perturbation, for (56) with $\beta>0$ and $\mathrm{Ri}=0.5$. The pressure field is asymmetric, due to the shape of the $p_{1}$ field, and its asymmetry bears some resemblance to that found in Fig. 6 . This is presumably due to the fact that, in both cases, the wind rotates anticlockwise as $z$ increases. However, the asymmetry does not cause here any misalignment of the drag with the surface wind, because both the maximum and the minimum of the pressure perturbation are displaced to the right of the surface wind by a similar amount. This configuration is caused by the $p_{1}$ field, which weakens the $p_{0}$ field in the first and second quadrants of the plot but reinforces it in the third and fourth. Figure $9 \mathrm{~b}$ shows that, although most of these qualitative features are confirmed by numerical simulations of the NH3D model, the pressure pattern is slightly rotated, suggesting a small drag component along $y$.

\section{d. Discussion}

The agreement of the present analytical model with the numerical simulations in the three examples treated above suggests that the pressure field at the surface is primarily influenced by the flow field near the surface and that, for example, critical levels play a relatively unimportant role in determining the surface drag, at least 

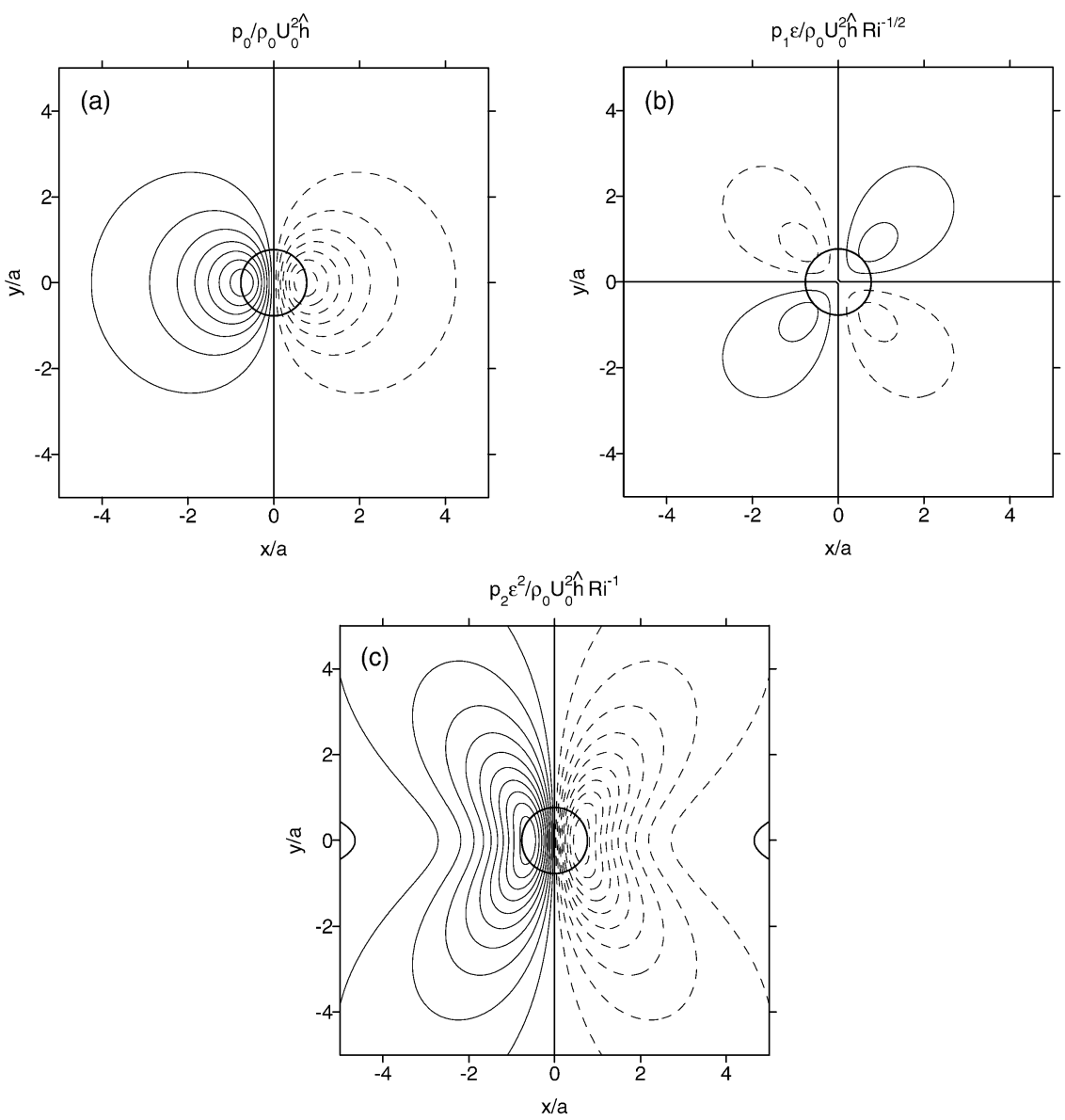

FIG. 8. Cross sections of the contributions to the normalized pressure perturbation at the surface given by the present model for the flow (56). (thick solid line) Terrain elevation equal to $0.5 h_{0}$; (solid contours) positive values; (dashed contours) negative values. (a) Zeroth-order pressure, contour spacing 0.05; (b) first-order pressure, contour spacing 0.025; and (c) second-order pressure, contour spacing 0.005 .
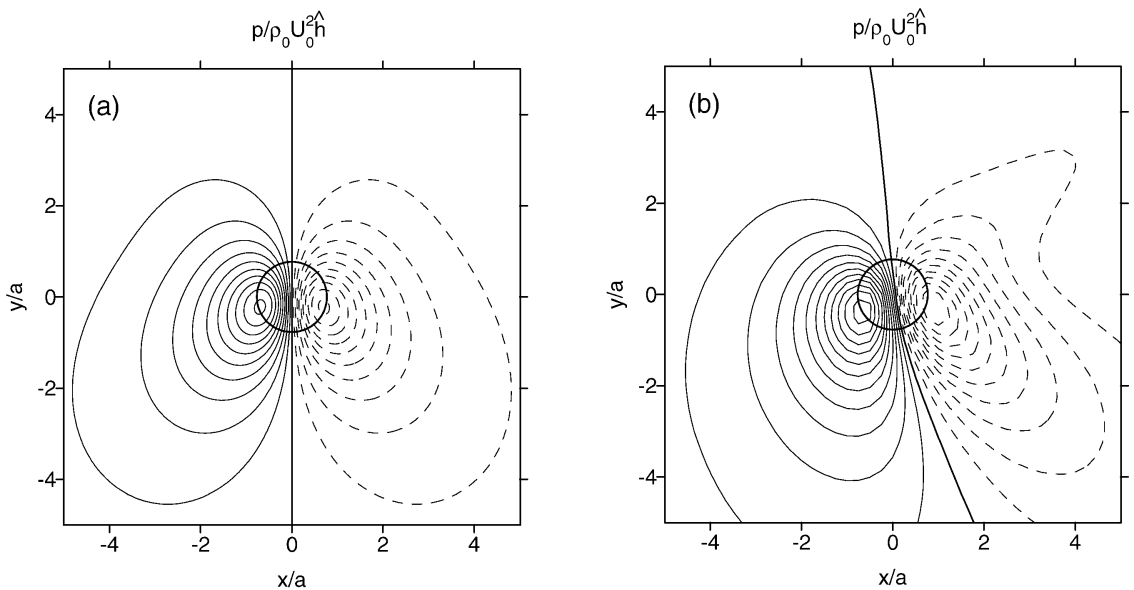

FIG. 9. Normalized total pressure perturbation at the surface for the flow given by (56) when $\mathrm{Ri}=0.5$. (thick solid line) Terrain elevation equal to $0.5 h_{0}$; (solid contours) positive values; (dashed contours) negative values, contour spacing 0.05. (a) Present model; and (b) NH3D numerical model. 
in the parameter regimes considered here. The pressure perturbation that causes the drag is given in general by a 3D Poisson equation that is obtained by taking the divergence of the momentum equation, and using mass conservation. This Poisson equation has a source term depending directly on the unperturbed velocity profile. Due to the properties of the Laplacian operator, the pressure perturbation strictly depends on the velocity field in the whole domain. The fact that, in the WKB approximation, the surface drag only depends on the characteristics of the incoming flow at the surface means that the pressure is determined locally when the wind changes sufficiently slowly with height. This raises two important questions. 1) How slow must this wind variation be? 2) What should, in a real situation, be taken as the "surface" velocity appropriate for using in an inviscid model such as the one developed here?

The answer to question 1 appears to be, not very slow, as is corroborated by the accuracy of the model predictions for Richardson numbers as small as 0.5. One crude explanation for the good performance of the model at $\mathrm{Ri}=O(1)$ is that the coefficients multiplying $\mathrm{Ri}^{-1}$ in the drag formulas are relatively small (e.g., 3/32, $1 / 32,5 / 32$ ), so the series expansion for the drag is asymptotic even when $\mathrm{Ri}$ is relatively small. More generally, previous studies have shown that the WKB approximation can in fact be applied to situations where it would not, in principle, be strictly valid (Grisogono 1995).

Regarding question 2, boundary layer theory suggests that the inviscid pressure is transmitted almost unaltered through the region where the surface velocity varies due to the no-slip boundary condition. With this reasoning, the velocity (and velocity derivatives) to use in the present model should perhaps be those in the middle or upper part of the atmospheric boundary layer. In their study of the effects of form drag versus gravity wave drag, Belcher and Wood (1996) suggest, more specifically, that the velocity to use in inviscid models should be that at the top of their "middle layer," which separates the region where shear (but not turbulence) is important from the region where the unperturbed flow is not only inviscid but also approximately irrotational. While Belcher and Wood's (1996) scaling analysis is useful for having an idea of the order of magnitude of this height, their treatment is limited, since they only take into account shear in a logarithmic surface layer, disregarding aspects such as the Ekman boundary layer.

The present study complements the study of Belcher and Wood (1996) by showing that shear outside the boundary layer has an important impact on the surface gravity wave drag. Of course, before the present model can be used in parameterizations of gravity wave drag in large-scale models, the height at which to evaluate the unperturbed velocity and its derivatives has to be defined more objectively. That is a suitable topic for future investigations.

\section{Conclusions}

An analytical linear model based on the WKB approximation has been developed to investigate the gravity wave drag exerted on mountains by stratified flows where the velocity changes relatively slowly with height. By extending the WKB approximation up to higher order than previously considered, general analytical formulas for the drag have been derived, in the hydrostatic approximation, that show the impact on the drag of the first and second derivatives of the wind velocity at the surface.

To test the drag expressions derived in the present study, three simplified situations were considered, all of them characterized by a Richardson number constant in height: 1) unidirectional shear flow consisting of wind with a linear variation, 2) directional shear flow consisting of wind where one of the velocity components varies linearly and the other is constant, and 3) directional shear flow consisting of wind that turns with height at a constant rate, maintaining its magnitude. In these three cases, the variation of the surface drag with the Richardson number was found to be proportional to $\mathrm{Ri}^{-1}$. For flow 1, the drag is aligned with the surface wind and given by $D_{0}[1-3 /(32 \mathrm{Ri})]$, where $D_{0}$ is the corresponding value in the absence of shear. In case 2 , where the surface wind is at an angle of $45^{\circ}$ to the $x$ and $y$ axes, the drag is given by $D_{x}=D_{0}[1-3 /(32 \mathrm{Ri})]$ and $D_{y}=D_{0}[1-1 /(32 \mathrm{Ri})]$. It is, therefore, not aligned with the surface wind, especially for relatively small Ri. In these two cases the drag decreases as Ri decreases. For flow 3, the drag increases as Ri decreases, being given by $D_{0}[1+5 /(32 \mathrm{Ri})]$, and is aligned with the surface wind. All these predictions are shown to be in fairly good agreement with data from numerical simulations of the nonlinear, nonhydrostatic equations of motion, especially for large values of $\mathrm{Ri}$, but also even for values of Ri of $O(1)$.

Cross sections of the pressure perturbation at the surface calculated using the linear model reveal the three leading-order contributions to the pressure and their effects on the surface drag. The zeroth-order contribution is equal to that valid for a wind profile with constant velocity; the first-order contribution is symmetric with respect to the mountain and therefore produces no drag. It is only the second-order contribution (proportional to $\mathrm{Ri}^{-1}$ ) that modifies the drag, either weakening or reinforcing the zeroth-order contribution. This explains why previous models based on the WKB approximation, which consider terms only up to first-order, fail to detect any differences between the drag for flow with shear and flow with a constant velocity.

In the hydrostatic approximation, the absolute value of the drag in the absence of shear depends on the shape of the orography. But the coefficients of the relative corrections due to the variation of the unperturbed flow do not, as long as the orography is axisymmetric. This happens because the expressions for the pressure at ze- 
roth-, first-, or second-order, are independent of the wavenumber magnitude. Hence, the formulas presented in this study for flows 1, 2, and 3 are valid for any axisymmetric mountain. This property does not hold for nonhydrostatic flows, because the terms associated with nonhydrostatic effects depend on the horizontal wavenumber of the orography.

A natural next step in the line of research initiated by this study is to consider nonhydrostatic effects, which are certainly important for sufficiently narrow mountains, and to extend the analysis to more complicated orography geometries [e.g., elliptically shaped mountains, following Phillips (1984)]. Such developments would facilitate the formulation of parameterization schemes, since in these schemes the anisotropy of the orography must generally be taken into account.

Acknowledgments. This work was supported by Fundação para a Ciência e Tecnologia (FCT) under project BULET/33980/99, cofinanced by the European Union under program FEDER. MACT acknowledges the financial support of FCT under Grant SFRH/BPD/3533/ 2000. Suggestions and comments made by B. Grisogono and two anonymous referees are also gratefully acknowledged.

\section{REFERENCES}

Bacmeister, J. T., and R. T. Pierrehumbert, 1988: On the high-drag states of nonlinear stratified flow over an obstacle. J. Atmos. Sci., 45, 63-80.

Belcher, S. E., and N. Wood, 1996: Form and wave drag due to stably stratified turbulent flow over low ridges. Quart. J. Roy. Meteor. Soc., 122, 863-902.

Bender, C. M., and S. A. Orszag, 1999: Advanced Mathematical Methods for Scientists and Engineers. Springer, 593 pp.

Broad, A. S., 1995: Linear theory of momentum fluxes in 3-D flows with turning of the mean wind with height. Quart. J. Roy. Meteor. Soc., 121, 1891-1902.

Clark, T. L., and W. R. Peltier, 1984: Critical level reflection and the resonant growth of nonlinear mountain waves. J. Atmos. Sci., 41, 3122-3134.

Durran, D. R., 1992: Two-layer solutions to Long's equation for vertically propagating mountain waves: How good is linear theory? Quart. J. Roy. Meteor. Soc., 118, 415-433.

Grisogono, B., 1994: Dissipation of wave drag in the atmospheric boundary layer. J. Atmos. Sci., 51, 1237-1243.
_ 1995: A generalized Ekman layer profile with gradually varying eddy diffusivities. Quart. J. Roy. Meteor. Soc., 121, 445-453.

Grubišić, V., and P. K. Smolarkiewicz, 1997: The effect of critical levels on 3D orographic flows: Linear regime. J. Atmos. Sci., 54, 1943-1960.

Huppert, H. E., and J. W. Miles, 1969: Lee waves in a stratified flow. Part 3. Semielliptical obstacle. J. Fluid Mech., 35, 481-496.

Keller, T. L., 1994: Implications of the hydrostatic assumption on atmospheric gravity waves. J. Atmos. Sci., 51, 1915-1929.

Lilly, D. K., and J. B. Klemp, 1979: The effects of terrain shape on nonlinear hydrostatic mountain waves. J. Fluid Mech., 95, 241261.

Long, R. R., 1953: Some aspects of the flow of stratified fluids, a theoretical investigation. Tellus, 5, 42-58.

McFarlane, N. A., 1987: The effect of orographically excited gravity wave drag on the general circulation of the lower stratosphere and troposphere. J. Atmos. Sci., 44, 1775-1800.

Miranda, P. M. A., 1991: Gravity waves and wave drag in flow past three-dimensional isolated mountains. Ph.D. thesis, University of Reading, $190 \mathrm{pp}$.

— the wave drag: Splitting flow and breaking waves. Quart. J. Roy. Meteor. Soc., 118, 1057-1081.

— dimensional flow past isolated mountains. J. Atmos. Sci., 54, $1574-1588$.

Phillips, D. S., 1984: Analytical surface pressure and drag for linear hydrostatic flow over three-dimensional elliptical mountains. $J$. Atmos. Sci., 41, 1073-1084.

Press, W. H., B. P. Flannery, S. A. Teukolsky, and W. T. Vetterling, 1992: Numerical Recipes in Fortran. Cambridge University Press, 992 pp.

Scinocca, J. F., and W. R. Peltier, 1991: On the Richardson number dependence of nonlinear critical-layer flow over localized topography. J. Atmos. Sci., 48, 1560-1572.

Shutts, G. J., 1995: Gravity-wave drag parameterization over complex terrain: The effect of critical-level absorption in directional windshear. Quart. J. Roy. Meteor. Soc., 121, 1005-1021.

__ 1998: Stationary gravity-wave structure in flows with directional wind shear. Quart. J. Roy. Meteor. Soc., 124, 1421-1442. -, and A. Gadian, 1999: Numerical simulation of orographic gravity waves in flows which back with height. Quart. J. Roy. Meteor. Soc., 125, 2743-2765.

Smith, R. B., 1979: The influence of the earth's rotation on mountain wave drag. J. Atmos. Sci., 36, 177-180.

, 1980: Linear theory of stratified hydrostatic flow past an isolated obstacle. Tellus, 32, 348-364.

— 1985: On severe downslope windstorms. J. Atmos. Sci., 42, 2597-2603.

- 1986: Further development of a theory of lee cyclogenesis. $J$. Atmos. Sci., 43, 1582-1602.

Smolarkiewicz, P. K., and R. Rotunno, 1989: Low-Froude number flow past three-dimensional obstacles. Part I: Baroclinically generated lee vortices. J. Atmos. Sci., 46, 1154-1164.

Valente, M. A., 2000: Effects of directional wind shear on orographic gravity wave drag. Ph.D. thesis, University of Reading, 140 pp. 